\section{Lenalidomide active in patients with refractory or relapsed chronic lymphocytic leukemia}

Chronic lymphocytic leukemia (CLL) is a disease characterized by an abnormal cytokine milieu that supports the survival of malignant cells and causes resistance to therapy. Limited treatment options are available for individuals with CLL, and patients eventually experience disease relapse. Lenalidomide is an immunomodulating drug that can induce apoptosis in malignant cells by the activation of $T$ cells and natural killer cells. A recent nonrandomized phase II study by Chanan-Khan et al. assessed the effects of lenalidomide in patients with CLL.

The study included 45 patients with relapsed or refractory B-cell CLL who received daily $25 \mathrm{mg}$ lenalidomide for 21 days. In general, lenalidomide was well tolerated; however, some patients experienced treatment-related toxicities, particularly fatigue $(83 \%)$ and flare reaction (58\%). Overall, major responses were seen in $47 \%$ of patients, with $9 \%$ achieving a complete response and $38 \%$ achieving a partial response. Antileukemic activity of lenalidomide was evident after 8 days of administration, with $70.5 \%$ of patients exhibiting decreased peripheral absolute lymphocyte counts.

This study is the first to demonstrate the antitumor activity of lenalidomide in CLL. The drug seems well-tolerated, with the only side effects being those commonly seen with other therapeutic options used for the treatment of CLL. On the basis of these results, the authors conclude that a large phase II study of lenalidomide is warranted.

Original article Chanan-Khan A et al. (2006) Clinical efficacy of lenalidomide in patients with relapsed or refractory chronic lymphocytic leukemia: results of a phase II study. J Clin Oncol 24: 5343-5349

\section{Study demonstrates clinical value of basal phenotype screening in breast cancer}

The basal phenotype (BP) of breast cancer is well recognized; however, the clinical significance of this class of breast cancer is not clearly understood. To address this situation, Rakha et al. examined a well-characterized series of 1,872 invasive breast carcinomas.
Breast cancer tissue microarrays were prepared and stained for basal cytokeratins (CK5/6 and/ or CK14). A tumor was defined as BP if basal cytokeratin expression was detected in $\geq 10 \%$ of invasive cells.

In total, the BP was identified in $18.6 \%$ of cases. Overall survival and disease-free interval were shorter in patients with BP tumors than in those with non-basal tumors, regardless of lymph node status. Stratification according to histological grade revealed BP as highly prognostic in grade 3 , but not in grade 1 or 2 , tumors. Indeed, BP was the most significant prognostic factor for overall and diseasefree survival in lymph-node-negative grade 3 tumors ( $P=0.003$ and $P<0.001$, respectively). The researchers also documented a trend towards improved outcome for patients with BP tumors who received adjuvant chemotherapy compared with those who did not; however, this observation requires further confirmation.

From these results, the authors conclude that $\mathrm{BP}$ is a valuable prognostic marker in breast cancer, identifying a subgroup of patients that might benefit from more-aggressive adjuvant therapy. They recommend routine screening of breast cancer for BP, particularly in patients with grade 3 tumors.

Original article Rakha EA et al. (2006) Basal phenotype identifies a poor prognostic subgroup of breast cancer of clinical importance. Eur J Cancer 42: 3149-3156

\section{C0X2 expression inhibits radiation-induced apoptosis}

The expression of cyclo-oxygenase 2 (COX2) has been documented in a variety of cancer tissues. In addition, a number of studies have linked the upregulation of COX2 to tumor growth and progression. Now, for the first time, Ishikawa et al. have clinically demonstrated that COX2 expression impairs the treatment effect of radiotherapy by inhibiting radiation-induced apoptosis.

In a prospective study, the researchers recorded the apoptotic index (Al) of cervical cancer biopsy specimens obtained from 47 consecutive patients before and 1 week after the initiation of radiotherapy. Of the 47 tumors examined, 22 were classed as COX2-positive (positive immunoreactivity accounting for $>50 \%$ of the specimen field examined). The mean 